Please quote as: Benner, D.; Schöbel, S. \& Janson, A. (2021): Exploring the State-ofthe-Art of Persuasive Design for Smart Personal Assistants. In: International Conference on Wirtschaftsinformatik (WI). Duisburg-Essen, Germany. 


\title{
Exploring the State-of-the-Art of Persuasive Design for Smart Personal Assistants
}

\author{
Dennis Benner ${ }^{1}$, Sofia Schöbel ${ }^{1}$, Andreas Janson ${ }^{2}$ \\ ${ }^{1}$ University of Kassel, Information Systems, Kassel, Germany \\ \{dennis.benner, sofia.schoebel\}@uni-kassel.de \\ ${ }^{2}$ University of St. Gallen, Institute of Information Management, St. Gallen, Switzerland \\ \{andreas.janson\}@unisg.ch
}

\begin{abstract}
Driven by technological advances, smart personal assistants (SPA) have gained importance in human-computer interaction. SPA can influence user behavior and persuade users to reach a specific outcome. However, users often lack the motivation to interact with SPA. One way to support this interaction is persuasive system design - considering concepts as gamification and nudging. Although SPA research has increased recently, there is still no shared knowledge about persuasive designs. Therefore, we aim to identify the current state-of-theart Design for persuasive SPA to understand how interactions and designs can be improved. Thus, we conduct a systematic literature analysis to represent how gamification and digital nudging are used to design SPA and conclude if and how those concepts can support SPA interactions. Consequently, we contribute to theory, providing better understanding about SPA interaction and design to make SPA more engaging and entertaining. Practitioners can use this contribution for persuasive SPA designs.
\end{abstract}

Keywords: Smart Personal Assistants, Persuasive Design, Persuasive Agents.

\section{Introduction}

With the increasing digitalization and influx of new information technologies, the impact of machines on people's lives is growing [1]. This growing impact extends to human decision-making, influencing human behavior as well as the interaction between humans and computers [2]. One trend that heavily impacts this human-computer interaction (HCI) is smart technical objects (STO). They are context-sensitive technical artifacts that use artificial intelligence (AI) and support (semi-)autonomous behavior to enhance the interaction [3]. STO include smart personal assistants (SPA), which are software programs that use AI and natural language to communicate with humans in a human-like fashion $[4,5]$. SPA fulfil tasks, provide assistance or smart services to users that change the user experience as well as personal satisfaction [6-8]. This technology offers various possible applications for research and practice, which have been successfully applied in the past $[9,10]$. For example, SPA are used in the context of ecommerce and customer service [7] because they can deliver a human-like experience that is always available [11]. These properties can positively transform the provider-

16th International Conference on Wirtschaftsinformatik,

March 2021, Essen, Germany 
user interaction and user experience permanently $[12,13]$, which may prove to be a key success factor for companies [14]. Consequently, the interest in SPA is expected to grow, as Gartner predicts that by 2020 up to $25 \%$ of all customer service operations will make use of SPA [15]. Therefore, SPA are expected to be the new standard path for users to interact with service providers [16]. Overall, the global market of SPA is expected to grow by $24,3 \%$ until 2025 . This equals a total market net worth of 1.25 billion US-Dollar [17].

However, SPA do have their share of problems. A recent survey on the interaction of users with an SPA shows that $58 \%$ of users could not complete their task (customer support inquiry), $52 \%$ did not like the interaction and $73 \%$ stated that they did not enjoy SPA in general [18]. Academic studies confirm these issues and highlight that users get demotivated because of bad SPA design, as Pricilla et al. [19] and Adam et al. [7] find in their studies in the field of e-commerce and self-service. These problems will eventually result in unsatisfactory experiences [7], bad performance and ultimately also to the failure of badly designed SPA [20]. Oftentimes, this bad design refers to the general ability to motivate users to engage and interact with the SPA as well as the ability to create joyful and satisfying user experiences. This shortcoming in current SPA design indicates that such designs lack the means for sustainable user motivation and engagement, thus missing to deeply consider the psychological needs of users and reducing effects on desired behavioral changes in users. This in return may lead to users not properly interacting with SPA, aborting their interaction and eventually rejecting SPA in total. Therefore, the need for an engaging and motivating SPA design arises that satisfies the needs of users and thus prevents failures [16,20].

Current literature regarding SPA design lacks knowledge about design elements and configurations [21], especially in the area of persuading users to motivate and engage them to change their behavior towards a desired goal. To solve this problem, it can be referred to concepts that engage users and guide them during the interaction with an SPA, combining motivating components with components that alter a user's behavior. In doing so, service providers can, for example, make users disclose more non-private information to the SPA with the ultimate goal of improving the interaction (e.g., providing better assistance or recommending better suited products to the customer).

Two related ways to implement such components is to refer to persuasive system designs, which considers gamification [22] and digital nudging [23]. Gamification in its essence is the use of game design elements in a non-game context [24] that can positively impact motivation and engagement as well as change behavior [25, 26]. Digital nudging (or simply "nudging"), on the other hand, simply makes use of small design elements that persuade humans to pursue a specific behavior [23]. This can be illustrated with an example: interacting with an SPA more effectively and thus receiving better service is in the interest of both user and service provider. Consequently, we propose the design idea of "persuasive smart personal assistants" (pSPA). The concept of pSPA relies on persuasive technology and design that motivates users (in a gameful fashion) and persuades (or nudges) them to change their behavior in favor of a desired outcome [27].

Therefore, we aim to combine the two related concepts of gamification and digital nudging in the effort to create a first pSPA concept in order to increase the motivation 
of users during the interaction with SPA. Thus, we seek to address the research gap on SPA design and answer the following two research questions (RQ):

RQ1: What is the state-of-the-art design for persuasive smart personal assistants?

\section{RQ2: How do current SPA incorporate persuasive design features?}

We will answer these two questions by conducting a systematic analysis of current literature [28-31]. Ultimately, our research will contribute to the knowledge base for SPA design by analyzing current SPA designs with persuasive features.

The structure of this research paper is as follows. First, the theoretical background for relevant topics, like SPA and persuasive system design, is explained. Then the research methodology is presented. Next, the results of the literature review are discussed. The paper then closes with a conclusion, implications of the results and the importance for future research as well as a planned research agenda.

\section{Theoretical Background}

\subsection{Smart Personal Assistants and User Interaction}

The term "Smart Personal Assistant" (SPA) is an umbrella term for technological artifacts (i.e., computer programs) that use voice, vision and contextual information to interact with humans [32]. The general idea behind SPA is a technology-based approach to fulfill tasks and to provide assistance for humans [3, 5, 33]. Modern SPA additionally make use of AI - including machine learning (ML) and natural language processing (NLP) - to interact with humans [4]. Such SPA are nowadays omnipresent, as in the Facebook messenger app [9].

In the narrow sense modern SPA are often defined as "smart voice assistants" and restricted to examples like Alexa or Siri. However, with this study, we want to analyze the state of the art of how to make user-SPA interactions more engaging to support users in changing their behavior. Consequently, in this research paper we use a broader definition that includes all kinds of assistants that often are used synonymously with "intelligent assistants", "virtual assistants" and "conversational agents". Such a broader definition also includes simple rule-based chatbots that may not be "smart" but may incorporate persuasive design features, which may prove to be useful for our planned contribution. Additionally, human-like artifacts such as robots will be included in this research, since the outcome of SPA and robots (i.e., embodied agents that may appear human-like) is the same [34]. We apply this broader scope to evaluate as many implementations and concepts - like gamification or digital nudging - as possible that may be applicable to our proposed persuasive design concept for smart personal assistants. Hence, the definition of SPA in this paper is any technological artifact that interacts in a human-like fashion with the user to fulfill a task or provide assistance.

Accordingly, SPA and users interact with each other. To better design interactive dialogues between a user and an SPA, they also incorporate techniques that include social norms and emotional aspects of interpersonal communication to address the psychological needs of users $[4,5,35]$. SPA imitate humans and try to address the 
psychological needs of humans, which are defined as the universal, basic needs inherent in every person. These needs are a driving factor behind engagement, motivation as well as an efficient and effective user-SPA interaction. If users are not satisfied, they get demotivated or frustrated easily [36].

\subsection{Persuasive System Design and Corresponding Concepts}

Persuasion can be defined as a form of communication with the intention to influence decisions and behaviors of people so that a desired outcome is achieved [37, 38]. Thus, the aim of persuasive technology and persuasive systems - henceforth Persuasive System Design (PSD) - is to influence users and their behavior to achieve a desirable outcome using persuasive design for information technology and systems [27]. To design persuasive technology or persuasive systems, the most prominent and effective concepts are gamification and digital nudging.

Gamification can be considered a persuasive technology that aims to exert influence over human behavior, which can be more powerful than monetary incentives [25]. A popular and widely accepted definition of gamification with a broad scope is the use of game design elements in a non-game context [24]. In addition to changing user behavior towards a desired outcome, gamification can provide a joyful experience and address hedonic aspects of products (i.e., services employing an SPA), which is significant for the user experience $[39,40]$.

Digital nudging, on the other hand, does not offer such joyful experiences but rather "nudges" humans towards a desired outcome [41]. Nudging, however, focuses on persuading people to change their behavior towards a desired outcome by using small design elements that influence the choice and behavior of people in a predictable way [23]. The main difference to gamification is that nudging explicitly affects user choice and must not forbid any options or change monetary incentives to achieve the desired behavior. However, both gamification and digital nudging are related and can support users in changing their behavior - or, more precisely, persuade users to reach a specific outcome a desired behavior.

Thus, we aim to highlight what similarities between gamification and digital nudging exist and how they can be applied to persuasive design for SPA. To compare and combine gamification and digital nudging, one must consider the different theoretical backgrounds and psychological needs these two persuasive methods employ. Despite the differences, gamification and nudging can be compared regarding their theoretical background [42]. An overlap regarding the psychological effects can be identified, which are (1) social norms, (2) priming, (3) motivation, (4) choice and (5) representation. Accordingly, gamification and digital nudging try to address the inner human needs of autonomy, relatedness and competence. This way, both gamification and digital nudging address similar basic psychological needs in human beings while also using similar theoretical backgrounds [36].

Apart from the shared goal of addressing psychological needs, gamification and nudging refer to design elements that instantiate the design of a persuasive technology. Gamification makes use of game design elements that address the psychological effects just mentioned. These design elements can be organized in a taxonomy along factors 
and attributes [43]. In our research we use the established game design element taxonomy introduced by Schöbel et al. [43]. On the one hand, factors represent the overarching category of design elements and the mechanic they use (e.g., progress, rewards and guidance). On the other hand, attributes express the actual implementations that refer to the overarching category (i.e., factors). For example, the attributes of points and badges refer to the factor rewards, while the attributes of feedback avatars and representing avatars refer to the factor guidance.

Similar to gamification, nudging also uses some specific design elements. However, unlike game design elements used in gamification that provide an established taxonomy, the categorization of design elements used for nudging is fairly new. Nevertheless, categorization is possible as Schöbel et al. [44] demonstrate. This taxonomy presents the following categories: default, representation and framing, information, feedback, time delay, social, and progress. The default nudge refers to default settings (e.g., cookie settings of a website). Representation and framing addresses visual factors like colors. Information and feedback nudges focus on providing knowledge, with information nudges being more general and feedback nudges being focused on a specific task or context. The time delay nudge is essentially equal to the time pressure game design element of gamification, limiting the user's time to make decisions. The social nudge refers to the user's social needs (e.g., social comparison), similar to how leaderboards address the need for social comparison in gamification. The progress element is also almost identical between nudging and gamification.

By comparing gamification and nudging design elements, a clear overlap between some elements can be observed. The most obvious overlapping design elements between gamification and nudging are time and progress, which are almost identical. Further, feedback is similarly defined in both gamification and nudging. However, gamification may focus more on visual feedback in this regard, while nudging may focus more on text-based feedback [43, 44]. Nevertheless, at the core both variants are identical in the sense that they keep the user aware about a circumstance or situation during the use of an information system [43, 44]. Apart from this overlap, some elements are unique to gamification and nudging, like gatherable points or badges to gamification and the default element to nudging.

With this background in mind, gamification and nudging can be combined in two ways. On the one hand, due to the existing overlap, the two concepts already support each other. On the other hand, both concepts provide exclusive design elements that can close the gaps between them and thus combine the advantages of mitigating possible disadvantages if applied on their own. For example, badges rely on a visual representation of the reward. This is not possible with voice assistants. Here, information and feedback nudges that mimic the content of the badge may be useful. This also highlights the importance of adjusting persuasive design elements to the properties of SPAs. Hence, gamification and digital nudging can both complement each other in addressing the psychological needs of users and translate them into a viable design for pSPA that are adjusted to the pSPA properties. In other words, persuasive design elements should be used according to the user-SPA interaction in question. Because the goal of this paper is to provide a broad view on options to apply PSD to 
SPA, restrictions like on digital nudging [23] are dropped. Consequently, we use the following definition for PSD in this research paper: Persuasive system design is the usage of any design element that exerts influence over users to change their behavior in a desirable fashion. To derive our concept of pSPA, we will take a closer look at what previous research studies have done to consider digital nudges and gamification in relation to SPA. Afterwards, we will try to find similarities between both concepts to better understand how we can support users with pSPA.

\section{$3 \quad$ Research Methodology}

To answer the research questions, a systematic literature review was conducted. The literature review was conducted according to the suggestions proposed by Cooper [28], Fettke [30] and Vom Brocke et al. [29]. A simplified process of our structured literature review that we adapted from Fettke [30] can be seen in Figure 1 below.

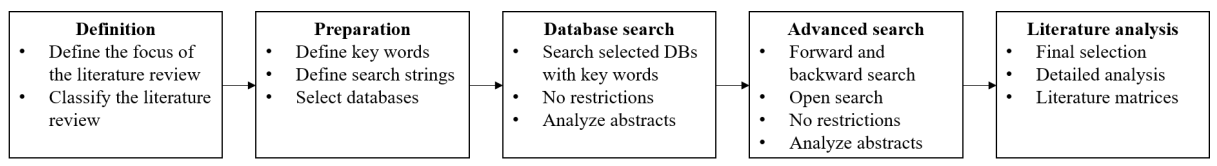

Figure 1. Structured Literature Review Process

Starting with the definition of our review, the goal was to explore the current stateof-the-art design options for pSPA. Hence, we focused on the integration of results with a focus on used design elements. The scope of the literature review was explorative so that the spectrum of state-of-the-art designs is as complete as possible. Accordingly, the structure was thematical and methodical.

\begin{tabular}{|l|l|l|l|}
\hline Characteristic & Category & \multicolumn{3}{|l|}{} \\
\hline Goal & Integration & Criticism & Central topics \\
\hline Scope & Representative & Selective & Explorative \\
\hline Focus & Outcomes & Designs & Theories \\
\hline Structure & Historical & Thematical & Methodical \\
\hline
\end{tabular}

Table 1. Literature Review Classification

The next step was the preparation of our literature search. Here, we defined necessary keywords and search strings that we used for our database search. Hence, we defined keywords that represent various forms and descriptions of SPA. These keywords included "virtual assistants", "smart assistants" and "conversational agents" as well as "gamification" and "nudging". Additionally, we also included the term "collaboration" as an optional keyword to highlight the collaborating factor in userSPA interaction. We did this because of the role of SPA in collaborative settings, where users and SPA create value together in smart services [3]. Accordingly, we formulated the following search string (optional search terms are in square brackets) that we adapted to the specifications of every database including the use of wildcards: 


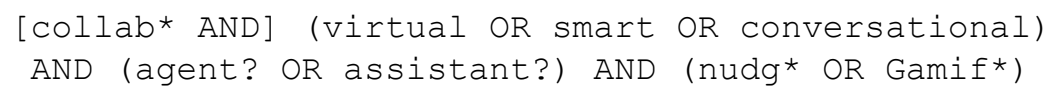

As for the databases, ScienceDirect, AISeL, IEEEXplore, EBSCOhost and ACM were included. Because of the novelty of this specific topic and the desired broad scope for the review, the literature was not subjected to any restrictions like publication date, peer-reviewed literature or ratings, and grey literature was considered as well, since it can provide value and broaden the horizon [45]. Additionally, we conducted a forward and backward as well as open search for further literature. A total of 5017 papers were found during the initial database literature search. Those papers were narrowed down to 691 papers, selected by title and abstract. Of those 691 papers, only 135 were selected for further reading after scanning their content. Those 135 papers were then examined thoroughly, and 23 papers remained and were found as relevant. These findings include forward and backward as well as open search. We based the inclusion or exclusion of studies on the implementation of gamification and nudging as well as the implemented artifact. Concerning the latter, we here referred to the taxonomy of Knote et al. [3]. However, we also included artifacts that do not fit to this taxonomy, based on the findings of Tussyadiah and Miller [34], who state that robotic artifacts are similar to virtual agents, which are defined in the taxonomy, and provide the same outcome. Concerning gamification and nudging, we included studies that apply to the definitions we describe in the theoretical background and excluded those that do not.

Consequently, the results of the gathered literature are then organized in concept matrices according to Webster and Watson to gain a deeper understanding of the composition of current persuasive design elements for SPA [31]. SPA come in different forms and shapes with various features; thus, SPA need to be categorized accordingly. To categorize SPA a simplified taxonomy by Knote et al. [3] is used, where only categories relevant to this topic are included, such as communication and representation. Literature that does not implement SPA but instead theorizes about SPA or implements related artifacts such as robots is listed in the "other" category. Gamification and digital nudging design elements will be categorized according to simplified taxonomies by Schöbel et al. [43, 44]. These taxonomies will be narrowed down to relevant categories and combined considering similarities and differences of design elements of gamification and digital nudging.

\section{$4 \quad$ Results}

The 23 analyzed papers show a heterogenous mixture of journal and conference contributions as well as gray literature. Unlike the composition, the publication dates are more homogenous; almost all publications are from the late 2010s with a few exceptions from the 2000s. We examined the 23 resulting papers regarding their implementation of SPA and use of persuasive design elements such as gamification and digital nudging. The detailed results regarding the composition of SPA and similar artifacts can be found in Table 2 (see 4.1). Detailed results regarding the composition of persuasive design elements can be found in Table 3 (see 4.2). 


\subsection{Status Quo of SPA Design with Persuasive Features}

The table below shows the status quo of the design of SPA that include persuasive design features from the literature we included. We used the taxonomy provided by Knote et al. [3] as a blueprint and simplified the taxonomy for our research.

\begin{tabular}{|c|c|c|c|c|c|c|c|c|c|c|c|c|c|c|}
\hline \multirow[b]{2}{*}{ Author (Year) } & \multicolumn{4}{|c|}{ : } & \multicolumn{2}{|c|}{ 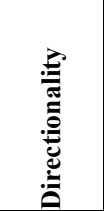 } & \multicolumn{2}{|c|}{ 总 } & \multicolumn{2}{|c|}{ 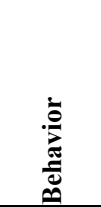 } & \multicolumn{3}{|c|}{ 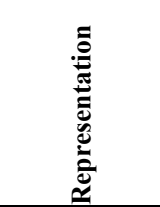 } & \multirow{2}{*}{ ప্ّ } \\
\hline & $\mathbf{T}$ & $\mathbf{A}$ & $\mathbf{V}$ & $\mathbf{P}$ & $\mathbf{U}$ & B & 1 & 2 & $\mathbf{S}$ & D & $\mathbf{N}$ & C & $\mathbf{O}$ & \\
\hline Backhaus et al. (2018) & & $\mathrm{X}$ & $\mathrm{X}$ & & & $\mathrm{X}$ & & & & & & $\mathrm{X}$ & $\mathrm{X}$ & $\mathrm{X}$ \\
\hline Brotman et al. (2015) & & & & $\mathrm{X}$ & & & & & & & & & & $\mathrm{X}$ \\
\hline Dokukina/Gumanova (2020) & $\mathrm{X}$ & & & & & & & & & & & & & $\mathrm{X}$ \\
\hline Eigenbrod/Janson (2018) & & & & & & & & & & & & & & $\mathrm{X}$ \\
\hline Falk et al. (2018) & $\mathrm{X}$ & & & & & $\mathrm{X}$ & & $\mathrm{X}$ & & $\mathrm{X}$ & $\mathrm{X}$ & & & \\
\hline Filimon et al. (2019) & & $\mathrm{X}$ & & & & $\mathrm{X}$ & & $\mathrm{X}$ & & $\mathrm{X}$ & $\mathrm{X}$ & & & \\
\hline Fischbach et al. (2018) & & $\mathrm{X}$ & $\mathrm{X}$ & & $\mathrm{X}$ & & $\mathrm{X}$ & & $\mathrm{X}$ & & & $\mathrm{X}$ & $\mathrm{X}$ & $\mathrm{X}$ \\
\hline Fogli et al. (2016) & $\mathrm{X}$ & & & & $\mathrm{X}$ & & $\mathrm{X}$ & & $\mathrm{X}$ & & & $\mathrm{X}$ & & $\mathrm{X}$ \\
\hline Hwang et al. (2019) & $\mathrm{X}$ & & & & $\mathrm{X}$ & & & $\mathrm{X}$ & $\mathrm{X}$ & & $\mathrm{X}$ & & & $\mathrm{X}$ \\
\hline Kocielnik et al. (2016) & & & & & & & & & & & & & & $\mathrm{X}$ \\
\hline Kuz et al. (2017) & $\mathrm{X}$ & & & & $\mathrm{X}$ & & $\mathrm{X}$ & & $\mathrm{X}$ & & & $\mathrm{X}$ & & \\
\hline Lange et al. (2020) & $\mathrm{X}$ & & & & $\mathrm{X}$ & & $\mathrm{X}$ & & $\mathrm{X}$ & & $\mathrm{X}$ & & & $\mathrm{X}$ \\
\hline Lechler et al. (2019) & $\mathrm{X}$ & & & & $\mathrm{X}$ & & $\mathrm{X}$ & & $\mathrm{X}$ & & $\mathrm{X}$ & & & \\
\hline Maedche et al. (2019) & & & & & & & & & & & & & & $\mathrm{X}$ \\
\hline $\begin{array}{l}\text { Martinez-Miranda et al. } \\
\text { (2008) }\end{array}$ & $\mathrm{X}$ & & $\mathrm{X}$ & & $\mathrm{X}$ & & $\mathrm{X}$ & & $\mathrm{X}$ & & & $\mathrm{X}$ & & $\mathrm{X}$ \\
\hline Sheth et al. (2019) & $\mathrm{X}$ & & & & $\mathrm{X}$ & & $\mathrm{X}$ & & $\mathrm{X}$ & & $\mathrm{X}$ & & & $\mathrm{X}$ \\
\hline Silva-Coira et al. (2016) & $\mathrm{X}$ & & & & & $\mathrm{X}$ & & $\mathrm{X}$ & & $\mathrm{X}$ & $\mathrm{X}$ & & & \\
\hline Smutny/Schreiberova (2020) & $\mathrm{X}$ & $\mathrm{X}$ & $\mathrm{X}$ & & & $\mathrm{X}$ & & $\mathrm{X}$ & & $\mathrm{X}$ & & $\mathrm{X}$ & $\mathrm{X}$ & $\mathrm{X}$ \\
\hline Strohmann et al. (2018) & & & & & & & & & & & & & & $\mathrm{X}$ \\
\hline Turk (2017) & & $\mathrm{X}$ & $\mathrm{X}$ & & & $\mathrm{X}$ & & $\mathrm{X}$ & & $\mathrm{X}$ & & $\mathrm{X}$ & $\mathrm{X}$ & $\mathrm{X}$ \\
\hline Tussyadiah/Miller (2019) & & & & & & & & & & & & & & $\mathrm{X}$ \\
\hline Weisz et al. (2019) & $\mathrm{X}$ & & & & & $\mathrm{X}$ & $\mathrm{X}$ & & $\mathrm{X}$ & & $\mathrm{X}$ & & & \\
\hline Xiao et al. (2019) & $\mathrm{X}$ & & & & & $\mathrm{X}$ & $\mathrm{X}$ & & $\mathrm{X}$ & & $\mathrm{X}$ & & & \\
\hline Sum (23) & 13 & 5 & 5 & 1 & 8 & 8 & 9 & 6 & 10 & 5 & 9 & 7 & 4 & 16 \\
\hline $\begin{array}{l}\mathrm{T}=\text { text; } \mathrm{A}=\text { audio/voice; } \mathrm{V}= \\
1 \text { = low complexity (rule-base } \\
\mathrm{S}=\text { static; } \mathrm{D}=\text { adaptive; } \mathrm{N}=\mathrm{n} \\
\text { Other = including non-SPA ar } \\
\text { design for SPA (e.g., future re }\end{array}$ & one; & $=\mathrm{v}$ & irtual & char: & 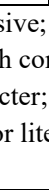 & $\mathrm{O}=$ & e sim & $\begin{array}{l}\text { /voice } \\
\text { ply th }\end{array}$ & only & & & ional & asive & \\
\hline
\end{tabular}

Table 2. Status Quo of Smart Personal Assistants with Persuasive Features 
We observed that most implementations in this study are mainly text-based (13 out of 23 studies), with a few exceptions that incorporate audio or voice (5) and visual forms (5) of communication. The interaction directionality (i.e., whether the interaction is one- or two-sided) is split relatively evenly ( 8 studies each). The complexity of the viewed SPA is also split with a slight tendency towards lesser complexity, with 9 studies choosing less complex and 6 studies opting for a more complex SPA design. This suggests that the current SPA design is built upon less complex methods like simple rule-based SPA or simple natural language applications in contrast to complex and advanced natural language processing and machine learning methods. SPA behavior is also tilted towards less complex static SPA in contrast to adaptive SPA (e.g., SPA that can be personalized), with 10 studies opting for static and only 5 for adaptive. The representation of SPA is mixed. Most text-based SPA do not use any form of representation (9), whereas SPA that use other forms of communication tend to have visual (7) and/or voice (4) representation. Additionally, it is noteworthy that many studies, regardless of whether they implement an actual SPA or not, at least indirectly acknowledge the existence of some persuasive influence in their study.

\subsection{Persuasive Design Elements from Literature}

A large portion of literature we analyzed does not directly implement an SPA. Instead, many authors rather chose to theorize about persuasive designs for SPA or simply highlighted persuasive effects they observed. Moreover, unlike the overall design of SPA with persuasive features as presented above, the use of design elements referring to digital nudging and gamification is less consistent, as can be seen in Table 2 above.

However, analyzing the designs of the included SPA, we can still identify a certain pattern. There is a clear tendency towards the use of information elements (14) exclusively from nudging and feedback elements (10), which exist in both gamification and nudging. Moreover, 10 studies also combine feedback and information elements (see [34, 46-54]), resulting in what we call an information-feedback pattern. However, looking at other elements from both nudging and gamification on their own, we observe a rather nonuniform distribution of several design elements. Some elements specifically from nudging - are missing entirely, like the default element to induce a desired behavior. Thus, we could not observe a clear tendency in design elements referring to gamification only, unlike with design elements that refer to digital nudging only (i.e., information elements). Regardless, game design elements are used for SPA to a certain degree. The most prominent observation of a game design element that exclusively refers to gamification is design elements that address the collection (5) mechanic. These include the collection of points or badges. Persuasive design elements that refer to both gamification and digital nudging, like design elements that address social norms and interactions, are being used as well. Studies use social comparison (3), as in rankings, and social collaboration (2), as in teamwork or trading things (e.g., virtual goods).

Additionally, it is noteworthy that some persuasive design elements are not being used at all. We could not observe design elements referring to progress (e.g., progress bars, levels) and default (e.g., default settings). 


\begin{tabular}{|c|c|c|c|c|c|c|c|c|c|c|}
\hline Author (Year) & $\mathbf{R}$ & $\mathbf{I}$ & $\mathbf{F}$ & $\mathbf{T}$ & $\mathbf{L}$ & $\mathbf{S}$ & $\mathbf{P}$ & $\mathbf{C}$ & $\mathbf{A}$ & $\mathbf{Z}$ \\
\hline Backhaus et al. (2018) & & & & & & & & $\mathrm{X}$ & & \\
\hline Brotman et al. (2015) & $\mathrm{X}$ & & $\mathrm{X}$ & $\mathrm{X}$ & & & & & & \\
\hline Dokukina/Gumanova (2020) & $\mathrm{X}$ & $\mathrm{X}$ & $\mathrm{X}$ & & & & & $\mathrm{X}$ & & \\
\hline Eigenbrod/Janson (2018) & & $\mathrm{X}$ & & & $\mathrm{X}$ & & & & & \\
\hline Falk et al. (2018) & & $\mathrm{X}$ & & & & & & & & \\
\hline Filimon et al. (2019) & & & & & $\mathrm{X}$ & & & $\mathrm{X}$ & & \\
\hline Fischbach et al. (2018) & & & & & & $\mathrm{X}$ & & & & \\
\hline Fogli et al. (2016) & & & & & & $\mathrm{X}$ & & $\mathrm{X}$ & & \\
\hline Hwang et al. (2019) & & $\mathrm{X}$ & $\mathrm{X}$ & & & & & & & \\
\hline Kocielnik et al. (2016) & & $\mathrm{X}$ & $\mathrm{X}$ & & & & & & & \\
\hline Kuz et al. (2017) & & & & & & & & & & $\mathrm{X}$ \\
\hline Lange et al. (2020) & & $\mathrm{X}$ & & & & & & & & \\
\hline Lechler et al. (2019) & $\mathrm{X}$ & $\mathrm{X}$ & $\mathrm{X}$ & & & & & & & \\
\hline \multicolumn{11}{|l|}{ Maedche et al. (2019) } \\
\hline Martinez-Miranda et al. (2008) & & & & & & & & $\mathrm{X}$ & $\mathrm{X}$ & \\
\hline Sheth et al. (2019) & & $\mathrm{X}$ & & & & & & & & \\
\hline Silva-Coira et al. (2016) & $\mathrm{X}$ & $\mathrm{X}$ & $\mathrm{X}$ & & & & & & & \\
\hline \multicolumn{11}{|l|}{ Smutny/Schreiberova (2020) } \\
\hline Strohmann et al. (2018) & & $\mathrm{X}$ & $\mathrm{X}$ & & & & & & & \\
\hline Turk (2017) & $X$ & $\mathrm{X}$ & $\mathrm{X}$ & & & & & & & \\
\hline Tussyadiah/Miller (2019) & & $\mathrm{X}$ & $\mathrm{X}$ & & $\mathrm{X}$ & & & & & \\
\hline Weisz et al. (2019) & & $\mathrm{X}$ & $\mathrm{X}$ & & & & & & & \\
\hline Xiao et al. (2019) & & $\mathrm{X}$ & $\mathrm{X}$ & & & & & & & \\
\hline $\begin{array}{ll}\text { Sum (23) } \\
\end{array}$ & 5 & 14 & 11 & 1 & 3 & 2 & 0 & 5 & 1 & 1 \\
\hline \multicolumn{11}{|c|}{$\begin{array}{l}\mathrm{R}=\text { representation (framing, avatars); } \mathrm{I}=\text { information (knowledge, hints); } \mathrm{F}=\text { feedback (response); } \\
\mathrm{T}=\text { time (pressure); } \mathrm{L}=\text { social comparison (leaderboards); } \mathrm{S}=\text { social collaboration (interaction, } \\
\text { exchange); } \mathrm{P}=\text { progress (levels, bars); } \mathrm{C}=\text { collection (points, badges); } \mathrm{A}=\text { aspiration (goals, quests); } \\
\mathrm{Z}=\text { gameful or playful design only }\end{array}$} \\
\hline
\end{tabular}

Table 3. State-of-the-Art Persuasive Design Elements from Literature

\subsection{Text-Based Information and Feedback Assistants}

In the results of our structured literature analysis we found a common design pattern among existing SPA. This pattern defined by its usage of persuasive design elements and general SPA properties. We define this pattern of text-based information and feedback assistants as TIFA, which can be considered a subcategory and/or design pattern of SPA. Artifacts that can be categorized as TIFA also show a tendency to unidirectional communication, static behavior and low complexity. This highlights the role of artifacts that fit the TIFA pattern. This approach to pSPA focuses on simple, unidirectional user-SPA interaction without adaptive behavior or advanced NLP/ML methods, which indicates some sort of "mentor" or "instructor" role of those pSPA. This TIFA pattern diverges from general observed SPA design in two ways. To 
illustrate how general SPA or pSPA compare to TIFA, we compare the concepts (see Figure 2; note that scales are relative and not an absolute representation of literature). First, TIFA are less complex regarding technology and implementation than regular SPA or other observed pSPA implementations. Second, TIFA focus entirely on two persuasive design elements - information and feedback. However, this does not imply TIFA cannot be supported by other persuasive design elements.
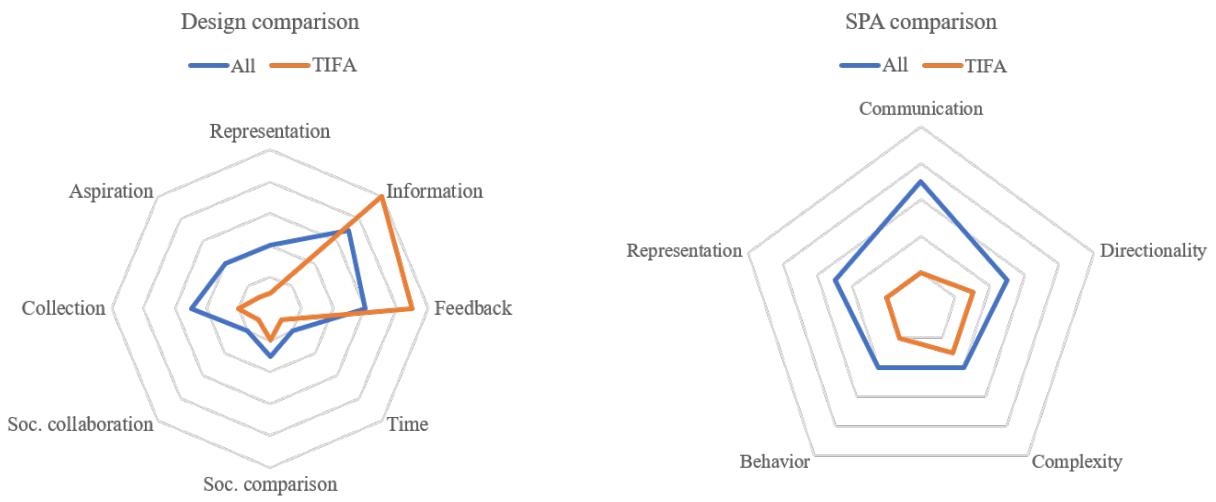

Figure 2.Comparison of observed SPA and TIFA pattern

We observed that some TIFA may implement design elements that use collection and social comparison, although these are not the norm for this pSPA pattern. The only true exception to this pattern is "ElliQ" [52], which is an embodied agent (e.g., robotic artifact) that combines information and feedback with focus on audiovisual interaction. This advanced artifact is also able to adapt to user behavior and the emotional state to provide adequate feedback.

\section{$5 \quad$ Discussion and Contributions}

\subsection{The State of SPA Research and Persuasive Design}

The goal of our systematic literature review was to provide an overview of designs with persuasive elements currently used in SPA research. In doing so, we answered two research questions with this study. Firstly, we want to stress that methodological research concerning a persuasive design for SPA is lacking and should be pursued in further research to gain more detailed knowledge. Secondly, we must clarify that some authors do not explicitly call their artifact an SPA or state that they implemented a persuasive design. Instead, some simply call their artifacts "agents" (e.g., [34, 55]) and/or highlight persuasive effects such as behavioral changes trough persuasive design elements (e.g., [34, 49, 55, 56]). Additionally, some other authors do not implement an SPA, persuasive design or other artifact at all. However, they do theorize about persuasive design regarding SPA. Many highlight the positive effects of designing the 
interaction in an engaging, game-like way, citing possible persuasive design elements derived from gamification such as rewards or reminders (e.g., [46, 50, 57-60]). Many other authors highlight the possible effects and uses of digital nudging for SPA by theorizing about its effects or implementation (e.g., [9, 34, 47-49, 51-54, 61-63]). Simply selecting and combining game design elements (or nudge elements respectively) does not necessarily support the intended behavioral change of users. There is some support in literature that one cannot refer to standardized solutions by simply integrating badges or points [64] to make an SPA interaction more entertaining. Likewise, when designing pSPA it needs to be considered how game design elements and nudge elements need to be designed and adapted to a context an SPA is interacting in. In general, authors that choose to focus on theorizing about the uses of persuasive design or effects on user behavior of SPA always include either gamification or digital nudging, with the latter being the prevalent theme. Authors repeatedly recognize the potential effects on user behavior and acknowledge future research potential regarding the application of gamification and/or digital nudging to SPA (e.g., [9, 64]).

In our study we find several persuasive design elements are being used that refer to the concepts of gamification and digital nudging. Some of them are used less frequently with no recognizable pattern, and others are used rather often with a recognizable pattern, often even combined. The prime example for this is the combination of the design elements of information and feedback [34, 46-54]. As described, many authors use those design elements either on their own or in combination. All studies, except one, that combine these elements use text-based SPA. This marks the greatest commonality that can be observed when analyzing current SPA that implement persuasive design elements. According to our literature analysis, this seems to be the go-to pattern for designing persuasive smart personal assistants. We emphasize on this pattern that we found in literature specifically, because of its prevalent usage over in multiple studies, where no other combination of design elements and SPA properties has shown usage to such an extent. However, this does not imply that there are no other patterns or combinations that may be relevant as well which other studies may find.

In general, many SPA designs incorporate persuasive elements, and authors use a persuasive design for their SPA, although most often only in an implicit way. In other words, many researchers do not explicitly apply persuasive design to their SPA but instead recognize the persuasive character and potential of their implementations retrospectively. This may point towards a lack of awareness about persuasive design and theory. On the other hand, authors are in fact aware of the effects. Considering the shortcomings in current SPA design and research as well as the highlighted potential of pSPA, we want to emphasize the importance of gaining awareness and consequently knowledge about this topic. Nevertheless, first approaches to designing persuasive agents or at least theorizing about persuasive effects of SPA designs that include gamification or digital nudging concepts can be found in the current literature. Another factor we want to highlight is that the majority of studies do not focus on the use of theories, psychological needs and effects or the preferences of the users but rather use ad hoc implementations of persuasive design. Another issue present in current SPA design research is the choice of persuasive design elements. While a common theme 
for information and feedback design elements can be observed, this cannot be said for other persuasive design elements.

Lastly, we want to state it may be valuable to examine progress, default and status quo design elements more closely to get an understanding why these are not used in the literature and may not work for pSPA design. In this regard, other studies may find different patterns similar to TIFA or entirely new ones in the future as research continues, designs change, and new artifacts are developed.

\subsection{Theoretical and Practical Contributions}

With our study, we provide implications for research and practice. Our research contributes to theory regarding general SPA interaction and design as well as theory about gamification and digital nudging. As a result of our systematic literature analysis, we identified the state-of-the-art persuasive design of today's SPA that is leaning towards SPA that primarily incorporate digital nudging and, to a certain degree, some game design elements. We introduce the TIFA pattern as a prevalent state-of-the-art theme for the design of current pSPA. We also identified several general shortcomings in current SPA design research and presented possible approaches to address those issues. Therefore, we contribute to theory by highlighting areas for future research about pSPA and about how to make a human-SPA interaction more meaningful. Practical implications can be given to developers of SPA about what to consider to better motivate and support users in an interaction with an SPA. From a practical perspective it is important to consider SPA designs that focus on how to better engage users in a SPA interaction to make it more effective and efficient for both the user and the constructor or a SPA (mainly a company or an institution). As a result, with our work, we provide a novel approach to practitioners about what to care about when constructing pSPA.

\section{Conclusion, Limitations and Future Research}

The goal of our paper was twofold. First, we wanted to provide an overview of the status quo of SPA design in order to describe a state-of-the-art design for persuasive smart personal assistants (RQ1). Second, we wanted to analyze how current SPA designs incorporate persuasive design features that refer to the concepts of gamification and nudging (RQ2). To address our research questions, we therefore conducted a structured literature review using established literature review methodology.

We presented theoretical studies and practical insights about the potential of SPA in general as well as the emerging opportunities for future research and practical applications of pSPA. In this regard we also presented a prevalent theme or pattern in current SPA with persuasive features that we define as TIFA. This pattern takes both technical properties of SPA as well as persuasive design features into account that refer to gamification and digital nudging concepts. Additionally, as a result of our structured literature review, we find that today there is virtually no methodical research that directly addresses persuasive design approaches for SPA. However, studies that use 
gamification and nudging in combination do acknowledge the persuasive character of their artifacts to a certain degree. However, we also have to address our own limitations in this regard. As with all literature-based research, our contribution as well is limited by our decisions to include or exclude certain studies, our definition of keywords and our search process. Moreover, the TIFA pattern we found in literature may or may not be supported by other, future studies based on the literature they include. As we earlier mentioned, we may have also missed other commonalities or themes in SPA research that refer to persuasive design. Disregarding potential limitations of our research, we in general want to highlight a potential research gap for the persuasive design of SPA that offers directions for future research. Future research should therefore focus on methodical design approaches, such as DSR, towards generalizable pSPA designs. Furthermore, conducting a meta-analysis on the topic of persuasive designs for SPA or pSPA designs may be a worthwhile proposition for future research.

Overall, based on our structured literature analysis we can summarize that explicit persuasive design in SPA research provides promising future research directions since many studies already acknowledge the implicit persuasive character of their SPA. We hope our contribution will encourage researchers to address the limitations and future directions that we presented.

\section{Acknowledgements}

The research presented in this paper was funded by European Regional Development Fund (ERDF) in the context of the project KI-based Subscription Business Automation (20007305), Grant No. pending. The project is conducted in cooperation with billwerk $\mathrm{GmbH}$ (see https://billwerk.com/forschungsprojekt-kiba/).

\section{References}

1. Bitkom e.V.: Die wichtigsten CRM-Trends im Check, https://www.bitkom.org/Themen/Technologien-Software/CRM/CRM-Trends-2017/3Chatbots.html

2. Skjuve, M., Haugstveit, I.M., Følstad, A., Brandtzaeg, P.B.: Help! Is my chatbot falling into the uncanny valley? An empirical study of user experience in human-chatbot interaction. Human Technology 15, 30-54 (2019)

3. Knote, R., Janson, A., Söllner, M., Leimeister, J.M.: Value Co-Creation in Smart Services: A Functional Affordances Perspective on Smart Personal Assistants. Journal of the Association for Information Systems (JAIS) (2020)

4. Guzman, A.: Making AI Safe for Humans: A Conversation With Siri. Socialbots and their friends: Digital media and the automation of sociality, 69-85 (2017)

5. Zhao, S.: Humanoid social robots as a medium of communication. New Media \& Society 8 , 401-419 (2006)

6. Medina-Borja, A.: Editorial Column-Smart Things as Service Providers: A Call for Convergence of Disciplines to Build a Research Agenda for the Service Systems of the Future. Service Science 7, ii-v (2015)

7. Adam, M., Wessel, M., Benlian, A.: AI-based chatbots in customer service and their effects on user compliance. Electron Markets (2020) 
8. Beverungen, D., Müller, O., Matzner, M., Mendling, J., Vom Brocke, J.: Conceptualizing smart service systems. Electron Markets 29, 7-18 (2019)

9. Maedche, A., Legner, C., Benlian, A., Berger, B., Gimpel, H., Hess, T., Hinz, O., Morana, S., Söllner, M.: AI-Based Digital Assistants. Bus Inf Syst Eng 61, 535-544 (2019)

10. Oracle: Can Virtual Experiences Replace Reality? The future role for humans in delivering customer experience (2019)

11. Meuter, M.L., Bitner, M.J., Ostrom, A.L., Brown, S.W.: Choosing among Alternative Service Delivery Modes: An Investigation of Customer Trial of Self-Service Technologies. Journal of Marketing 69, 61-83 (2005)

12. Forbes: AI And Chatbots Are Transforming The Customer Experience, https://www.forbes.com/sites/shephyken/2017/07/15/ai-and-chatbots-are-transforming-thecustomer-experience/

13. Reddy, T.: How chatbots can help reduce customer service costs by $30 \%$, https://www.ibm.com/blogs/watson/2017/10/how-chatbots-reduce-customer-service-costsby-30-percent/

14. Lemon, K.N., Verhoef, P.C.: Understanding Customer Experience Throughout the Customer Journey. Journal of Marketing 80, 69-96 (2016)

15. Gartner: Gartner Says 25 Percent of Customer Service Operations Will Use Virtual Customer Assistants by 2020, https://www.gartner.com/en/newsroom/press-releases/201802-19-gartner-says-25-percent-of-customer-service-operations-will-use-virtual-customerassistants-by-2020

16. Følstad, A., Brandtzæg, P.B.: Chatbots and the new world of HCI. interactions 24, 38-42 (2017)

17. Business Insider: Chatbot Market Size to Reach $\$ 1.25$ Billion by 2025 | CAGR: $24.3 \%$ : Grand View Research, Inc. | Markets Insider

18. Koelwel, D.: Chatbots im E-Commerce: Entwicklung eilt der Akzeptanz voraus, https://www.estrategy-magazin.de/e-commerce/artikel/chatobots-im-e-commerceentwicklung-eilt-der-akzeptanz-voraus-001543.html

19. Pricilla, C., Lestari, D.P., Dharma, D.: Designing Interaction for Chatbot-Based Conversational Commerce with User-Centered Design. In: 5th International Conference on Advance Informatics: Concepts, Theory and Applications. ICAICTA, August 14-17, 2018, Beyond Resort, Krabi, Thailand, pp. 244-249. IEEE, Piscataway, NJ (2018)

20. Brandtzaeg, P.B., Følstad, A.: Chatbots: changing user needs and motivations. interactions 25, 38-43 (2018)

21. Følstad, A., Brandtzaeg, P.B.: Users' experiences with chatbots: findings from a questionnaire study. Qual User Exp 5 (2020)

22. Morana, S., Friemel, C., Gnewuch, U., Maedche, A., Pfeiffer, J.: Interaktion mit smarten Systemen - Aktueller Stand und zukünftige Entwicklungen im Bereich der Nutzerassistenz. Wirtsch Inform Manag 9, 42-51 (2017)

23. Thaler, R.H., Sunstein, C.R.: Nudge. Improving decisions about health, wealth, and happiness. Yale Univ. Press, New Haven, Conn. (2008)

24. Deterding, S., Dixon, D., Khaled, R., Nacke, L.: From Game Design Elements to Gamefulness: Defining "Gamification". Proceedings of the 15th International Academic MindTrek Conference Envisioning Future Media Environments, 9-15 (2011)

25. Blohm, I., Leimeister, J.M.: Gamification. Bus Inf Syst Eng 5, 275-278 (2013)

26. Deterding, S.: Situated motivational afforances of game elements: A conceptual model. Computer Human Interfaces (2011)

27. Fogg, B.J.: Computers as persuasive social actors. In: Persuasive Technology, pp. 89-120. Elsevier (2003) 
28. Cooper, H.M.: Organizing knowledge syntheses: A taxonomy of literature reviews. Knowledge in Society 1, 104-126 (1988)

29. Vom Brocke, J., Riemer, K., Plattfaut, R.: Standing on the Shoulders of Giants: Challenges and Recommendations of Literature Search in Information Systems Research. CAIS 37 (2015)

30. Fettke, P.: State-of-the-Art des State-of-the-Art. Wirtsch. Inform. 48, 431 (2006)

31. Webster, J., Watson, R.T.: Analyzing the Past to Prepare for the Future: Writing a literature Review. MIS Quarterly, 13-21 (2002)

32. McTear, M., Callejas, Z., Griol, D.: The conversational interface. Talking to smart devices. Springer, Cham (2016)

33. Hauswald, J., Laurenzano, M.A., Zhang, Y., Yang, H., Kang, Y., Li, C., Rovinski, A., Khurana, A., Dreslinski, R.G., Mudge, T., et al.: Designing Future Warehouse-Scale Computers for Sirius, an End-to-End Voice and Vision Personal Assistant. ACM Trans. Comput. Syst. 34, 1-32 (2016)

34. Tussyadiah, I., Miller, G.: Nudged by a robot: Responses to agency and feedback. Annals of Tourism Research 78 (2019)

35. Skalski, P., Tamborini, R.: The Role of Social Presence in Interactive Agent-Based Persuasion. Media Psychology 10, 385-413 (2007)

36. Deci, E.L., Ryan, R.M.: The "What" and "Why" of Goal Pursuits: Human Needs and the Self-Determination of Behavior. Psychological Inquiry 11, 227-268 (2000)

37. Simons, H.W., Morreale, J., Gronbeck, B.E.: Persuasion in society. SAGE, Thousand Oaks, Calif. (2001)

38. Briñol, P., Petty, R.E.: Chapter 2 Persuasion. In: Zanna, M.P. (ed.) Advances in experimental social psychology, 41, pp. 69-118. Academic Press, London, Amsterdam, Oxford, Burlington, MA, San Diego, Calif. (2009)

39. Zhou, H., Fu, X.: Understanding, Measuring, and Designing User Experience: The Causal Relationship between the Aesthetic Quality of Products and User Affect. In: Proceedings of the 12th International Conference on Human-Computer Interaction: Interaction Design and Usability, pp. 340-349. Springer-Verlag, Berlin, Heidelberg (2007)

40. Sharp, H., Preece, J., Rogers, Y.: Interaction design. Beyond human-computer interaction (2019)

41. Acquisti, A., Sleeper, M., Wang, Y., Wilson, S., Adjerid, I., Balebako, R., Brandimarte, L., Cranor, L.F., Komanduri, S., Leon, P.G., et al.: Nudges for Privacy and Security. ACM Comput. Surv. 50, 1-41 (2017)

42. Lieberoth, A., Holm Jensen, N., Bredahl, T.: Selective psychological effects of nudging, gamification and rational information in converting commuters from cars to buses: A controlled field experiment. Transportation Research Part F: Traffic Psychology and Behaviour 55, 246-261 (2018)

43. Schöbel, S., Ernst, S.-J., Söllner, M., Leimeister, J.M.: More than the Sum of Its Parts Towards Identifying Preferred Game Design Element Combinations in Learning Management Systems. short paper. International Conference on Information Systems (2017)

44. Schöbel, S., Barev, T.J., Janson, A., Hupfeld, F., Leimeister, J.M.: Understanding User Preferences of Digital Privacy Nudges - A Best-Worst Scaling Approach. Hawaii International Conference on System Sciences, 3918-3927 (2020)

45. Mahood, Q., van Eerd, D., Irvin, E.: Searching for grey literature for systematic reviews: challenges and benefits. Research synthesis methods 5, 221-234 (2014)

46. Dokukina, I., Gumanova, J.: The rise of chatbots - new personal assistants in foreign language learning. Procedia Computer Science 169, 542-546 (2020) 
47. Hwang, I., Lee, Y., Yoo, C., Min, C., Yim, D., Kim, J.: Towards Interpersonal Assistants: Next-Generation Conversational Agents. IEEE Pervasive Comput. 18, 21-31 (2019)

48. Kocielnik, R., Xiao, L., Avrahami, D., Hsieh, G.: Reflection Companion: A Conversational System for Engaging Users in Reflection on Physical Activity. Proc. ACM Interact. Mob. Wearable Ubiquitous Technol. 2, 1-26 (2018)

49. Lechler, R., Stoeckli, E., Rietsche, R., Uebernickel, F.: LOOKING BENEATH THE TIP OF THE ICEBERG: THE TWO-SIDED NATURE OF CHATBOTS AND THEIR ROLES FOR DIGITAL FEEDBACK EXCHANGE. European Conference on Information Systems (ECIS) (2019)

50. Silva-Coira, F., Cortinas, A., Pedeira, O.: INTELLIGENT VIRTUAL ASSISTANT FOR GAMIFIED ENVIRONMENTS. Pacific Asia Conference on Information Systems (2016)

51. Strohmann, T., Fischer, S., Siemon, D., Brachten, F., Lattemann, C.: Virtual Moderation Assistance: Creating Design Guidelines for Virtual Assistants Supporting Creative Workshops. Pacific Asia Conference on Information Systems (2018)

52. Turk, V.: Home robot helps to keep you on your toes. New Scientist 233, 9 (2017)

53. Weisz, J.D., Jain, M., Joshi, N.N., Johnson, J., Lange, I.: BigBlueBot: Teaching Strategies for Successful Human-Agent Interactions. IUI 19, 448-459 (2019)

54. Xiao, Z., Zhou, M.X., Fu, W.-T.: Who should be my teammates: Using A Conversational Agent to Understand Individuals and Help Teaming. IUI 19, 437-447 (2019)

55. Brotman, R., Burleson, W., Forlizzi, J., Heywood, W., Lee, J.: Building Change:Constructive Design of Smart Domestic Environments for Goal Achievement. In: Begole, B., Kim, J., Inkpen, K., Woo, W. (eds.) Proceedings of the 33rd Annual ACM Conference on Human Factors in Computing Systems - CHI '15, pp. 3083-3092. ACM Press, New York, New York, USA (2015)

56. Falk, J., Poulakos, S., Kapadia, M., Sumner, R.W.: PICA: Proactive Intelligent Conversational Agent for Interactive Narratives. Proceedings of the 18th IVA, 141-146 (2018)

57. Filimon, M., Iftene, A., Trandabăț, D.: Bob - A General Culture Game with Voice Interaction. Procedia Computer Science 159, 323-332 (2019)

58. Fogli, D., Lanzilotti, R., Piccinno, A., Tosi, P.: AmI@Home: A Game-Based Collaborative System for Smart Home Configuration. AVI, 308-309 (2016)

59. Martinez-Miranda, J., Bernhard, J., Payr, S., Petta, P.: The Intermediary Agent's Brain: Supporting Learning to Collaborate at the Inter-Personal Level. Proc. of 7th Int. Conf. on Autonomous Agents and Multiagent Systems, 1277-1280 (2008)

60. Smutny, P., Schreiberova, P.: Chatbots for learning: A review of educational chatbots for the Facebook Messenger. Computers \& Education 151 (2020)

61. Eigenbrod, L., Janson, A.: How Digital Nudges Influence Consumers - Experimental Investigation in the Context of Retargeting. European Conference on Information Systems (ECIS) (2018)

62. Sheth, A., Yip, H.Y., Shekarpour, S.: Extending Patient-Chatbot Experience with Internetof-Things and Background Knowledge: Case Studies with Healthcare Applications. IEEE Intell. Syst. 34, 24-30 (2019)

63. Lange, P. de, Goschlberger, B., Farrell, T., Neumann, A.T., Klamma, R.: Decentralized Learning Infrastructures for Community Knowledge Building. IEEE Trans. Learning Technol., 1 (2020)

64. Liu, D., Santhanam, R., Webster, J.: Toward Meaningful Engagement: A Framework for Design and Research of Gamified Information Systems. MIS Quarterly 41, 1011-1034 (2017) 